Revista Española de Nutrición Humana y Dietética

www.elsevier.es/dietetica

\title{
REVISIÓN
}

\section{Diámetro abdominal sagital: aplicaciones en la práctica clínica}

\section{Thaís Da Silva Ferreira ${ }^{a}$, Gabriela De Azevedo Abreu ${ }^{\mathrm{b}}$, Marcelly Cunha Oliveira Dos Santos Lopes ${ }^{a}$, Vanessa Chaia Kaippert ${ }^{a}$ y Eliane Lopes Rosado ${ }^{a, *}$}

\author{
a Instituto de Nutrição Josué de Castro, Universidade Federal do Rio de Janeiro, Brasil \\ ${ }^{\mathrm{b}}$ Instituto de Estudos em Saúde Coletiva, Universidade Federal do Rio de Janeiro, Brasil
}

Recibido el 29 de diciembre de 2011; aceptado el 30 de octubre de 2012

\section{PALABRAS CLAVE \\ Grasa abdominal; \\ Diámetro abdominal \\ sagital; \\ Antropometría}

\section{KEYWORDS}

Abdominal fat;

Sagittal abdominal

diameter;

Anthropometry

\begin{abstract}
Resumen
El exceso de tejido adiposo visceral se asocia con factores de riesgo cardiovascular. Recientemente, el diámetro abdominal sagital (DAS) se ha convertido en un indicador de obesidad central y puede ayudar en la predicción del riesgo cardiovascular. El objetivo del estudio es revisar la literatura científica sobre la utilización del DAS para evaluación nutricional de adultos. Se llevó a cabo una búsqueda de artículos científicos en las bases de datos electrónicas SciELO , MEDLINE (PubMed) y Biblioteca Virtual de Salud. El DAS se asocia con la grasa abdominal (especialmente visceral) y diferentes factores de riesgo cardiovascular, como resistencia a la insulina, presión arterial y lipoproteínas plasmáticas, en mayor magnitud que indicadores tradicionales como el índice de masa corporal y la relación cintura/cadera. El DAS se puede utilizar en combinación con otras medidas antropométricas. Todavía no hay puntos de corte establecidos para la clasificación. El DAS puede ser una alternativa para estimar la grasa visceral, pero el pequeño número de estudios y la falta de consenso sobre la referencia anatómica para el registro de medidas dificultan la determinación del punto de corte para su interpretación.

(C) 2012 Asociación Española de Dietistas-Nutricionistas. Publicado por Elsevier España, S.L. Todos los derechos reservados.
\end{abstract}

\section{Sagittal Abdominal Diameter: Application in Clinical Practice}

\begin{abstract}
Excess visceral fat is associated with cardiovascular risk factors. Sagittal abdominal diameter (SAD) has recently been highlighted as an indicator of abdominal obesity, and also may be useful in predicting cardiovascular risk. The purpose of the present study was to review the scientific literature on the use of SAD in adult nutritional assessment. A search was conducted for scientific articles in the following electronic databases: SciELO
\end{abstract}

\footnotetext{
*Autor para correspondencia.

Correo electrónico: elianerosado@nutricao.ufrj.br (E. Lopes Rosado).
} 
, MEDLINE (PubMed) and Virtual Health Library. SAD is more associated with abdominal fat (especially visceral), and with different cardiovascular risk factors, such as, insulin resistance, blood pressure, and serum lipoproteins than the traditional methods of estimating adiposity, such as body mass index and waist-to-hip ratio. SAD can also be used in association with other anthropometric measures. There are still no cut-off limits established to classify SAD as yet. SAD can be an alternative measure to estimate visceral adiposity. However, the few studies on this diameter, and the lack of consensus on the anatomical site to measure SAD, are obstacles to establish cut-off limits to classify it. opulation studied developed DM2 or IFG. These diagnoses were related with IR and PFBC. ๑ 2012 Asociación Española de Dietistas-Nutricionistas. Published by Elsevier España, S.L. All rights reserved.

\section{Introducción}

Se ha asociado la acumulación de tejido adiposo (TA) visceral con enfermedades crónicas y trastornos metabólicos como hipertensión arterial (HTA) sistémica, resistencia a la insulina (RI), diabetes mellitus (DM) tipo 2 y dislipidemias ${ }^{1}$. Por lo tanto, es creciente el interés en la evaluación de la distribución de grasa corporal y la cuantificación de la adiposidad intraabdominal ${ }^{2}$.

Se han empleado diferentes parámetros antropométricos para medir la acumulación de TA. Para evaluar la obesidad generalizada, el índice de masa corporal (IMC) es el indicador más utilizado. Como indicador de obesidad central, se destaca el perímetro de la cintura (PC), ampliamente utilizado en estudios y la práctica clínica ${ }^{3}$. También se ha medido la distribución de la grasa corporal por índices como la razón perímetro de la cintura/perímetro de cadera (RCC), la razón $\mathrm{PC} /$ perímetro del muslo (PC/Pmuslo), la razón perímetro del cuello/Pmuslo (Pcuello/Pmuslo), el índice de conicidad (ICo $=$ PC / 1,109 Jpeso / talla) y la razón PC/talla $(\mathrm{RCT})^{4}$.

La tomografía computarizada (TC) y la resonancia magnética (RM) son los métodos más precisos para la evaluación del TA intraabdominal, pero no se suele utilizar estas pruebas en la práctica clínica debido a su alto coste, el largo tiempo de ejecución y la necesidad de equipo especializa$\mathrm{do}^{5}$. Para la investigación clínica y epidemiológica, se han utilizado más las medidas antropométricas, aunque estiman la grasa visceral de manera indirecta6.

Recientemente, varios autores han propuesto el uso del diámetro abdominal sagital (DAS) como un indicador de la adiposidad abdominal ${ }^{7-15}$.

El DAS representa la talla abdominal incluyendo la distancia entre la espalda y el abdomen. Esta medición se puede realizar con la ayuda de un compás o calibre o por técnicas de imagen como TC, RM o ultrasonidos (US) ${ }^{9,10,16,17 . ~}$

El DAS ha llamado la atención de la comunidad científica, especialmente después de los estudios de Henry Kahn, uno de los pioneros en la aplicación de esta medida para predecir el riesgo de morbilidad y mortalidad cardiovascula$\operatorname{res}^{18,19}$

El objetivo de este trabajo es revisar la literatura científica sobre la utilización del DAS para la evaluación nutricional de adultos en la práctica clínica.

\section{Métodos}

En el periodo de agosto a octubre de 2010, se hizo una búsqueda de estudios científicos en las bases de datos electrónicas: MEDLINE (vía PubMed), SciELO, Scopus y Biblioteca Virtual en Salud (BVS). Inicialmente, se buscó los descriptores más apropiados para el tema en el Medical Subject Headings (MeSH) y los Descriptores en Ciencias de la Salud (DeCS). No se encontraron los descriptores para esta medición antropométrica, por lo que se utilizaron términos libres para la realización de la búsqueda. Las palabras clave utilizadas en portugués fueron "diámetro abdominal sagital" OR diámetro abdominal sagital. En inglés, se usó el término "sagittal abdominal diameter" OR sagittal abdominal diameter. Se consideraron los artículos publicados en cualquier año.

No se establecieron restricciones en el idioma de los artículos o las características de la población: etnia, sexo o estado de salud. Se excluyeron los estudios realizados con niños y adolescentes. Después de la búsqueda, se seleccionaron los estudios que contenían protocolos de medición, puntos de corte propuestos y relación del DAS con otras variables antropométricas y/o resultados clínicos relevantes.

\section{Resultados y discusión}

\section{Importancia y aplicación del DAS para evaluación del estado nutricional}

La propuesta de utilización del DAS en la práctica clínica y estudios científicos como una medida alternativa para evaluar la obesidad abdominal se debe a su asociación con la grasa abdominal (especialmente la visceral) y con diferentes factores de riesgo cardiovascular ${ }^{2}$.

A pesar del consenso sobre las limitaciones de los parámetros antropométricos para la medición precisa de la grasa visceral, PC y DAS se correlacionan mejor con la grasa visceral que los métodos tradicionalmente utilizados, como IMC y RCC ${ }^{2}$

La utilización de los diámetros abdominales como indicadores de grasa visceral se investigó en un estudio longitudinal de Van Der Kooy et $\mathrm{al}^{10}$, en el cual evaluaron a 96 adul- 
tos obesos aparentemente sanos de ambos sexos. Los resultados indican que el DAS puede tener ventajas sobre PC y RCC en los varones, pero no en las mujeres.

Se ha estudiado la relación entre DAS y resultados clínicos relevantes como la $\mathrm{RI}^{4,20-22}$, las concentraciones de lipoproteínas aterogénicas elevadas ${ }^{23,24}$, las enfermedades cardiovasculares $(E C V)^{18,25,26}$, el síndrome metabólico $(S M)^{12}$ e incluso la disfunción eréctil ${ }^{27}$.

En un estudio transversal realizado en una muestra heterogénea de 157 mujeres (inmigrantes suecas y residentes nativas de un pueblo en Suecia), entre las cuatro variables antropométricas evaluadas (DAS, IMC, PC y RCC), el DAS fue la que presentó mayor correlación con la RI (evaluada por Homeostasis Model Assessment of Insulin Resistance [HOMA-IR]; $r=0,48 ; p<0,0001)$, los triglicéridos $(r=0,54$; $p<0,0001)$ y la proteína $C$ reactiva $(r=0,50 ; p<0,0001)$, lo que indica que el DAS podría ser un marcador clínico útil para estos factores de riesgo cardiovascular. Los resultados de los análisis multivariable que incluyen DAS, IMC y PC demuestran que solamente el DAS permanece como predictor significativo de RI ( $p=0,007)$, HOMA-IR $(p=0,001)$, triglicéridos $(p=0,009)$ y proteína $C$ reactiva $(p=0,008)^{20}$.

Vasques et $\mathrm{al}^{22}$, mediante estudio transversal, evaluaron la efectividad de los siguientes indicadores antropométricos en la identificación de RI en varones adultos brasileños $(\mathrm{n}=138)$ : DAS, PC, perímetro de la cadera (PCad), Pmuslo, ICo, IMC, índice sagital (IS = DAS / Pmuslo), RCT, RCC y PC / Pmuslo. DAS $(r=0,482)$ y PC $(r=0,464)$ presentaron mayor correlación con HOMA-IR y mayor capacidad de discriminación de RI $(p<0,001)$.

Con los resultados del Los Angeles Aterosclerosis Study (estudio longitudinal que involucró una muestra de 573 sujetos), Reed et al observaron una asociación significativa del DAS y el IMC con presión arterial, lipoproteínas plasmáticas, insulina en ayunas y peso corporal en adultos estadounidenses de mediana edad ${ }^{23}$.

La asociación del DAS con lipoproteínas plasmáticas también se describe en estudio transversal realizado en 260 varones estadounidenses y 282 japoneses. Los autores concluyeron que DAS y PC se asociaban significativa y positivamente con las concentraciones de partículas de lipoproteínas de muy baja densidad (VLDL) y de baja densidad (LDL), incluso después de ajustar por el IMC ${ }^{24}$. En ese estudio, los autores concluyeron que el DAS puede ser mejor predictor del riesgo de ECV que el PC, debido a que se correlaciona más con la grasa visceral, probablemente por el desplazamiento de la grasa subcutánea a los lados de la cintura durante la medición del DAS en posición supina ${ }^{24}$.

Valsamakis et $\mathrm{al}^{12}$, en un estudio transversal, investigaron qué medidas antropométricas (IMC, PC, PCad, RCT, RCC y DAS) estaban más relacionadas con SM en varones adultos $(n=83)$. El análisis de regresión múltiple mostró que DAS y PC son los mejores predictores de SM.

El DAS también podría reemplazar al PC para la evaluación de la obesidad relacionada con el riesgo cardiovascular. Se ha descrito que el DAS medido por imágenes de RM se asocia con SM independientemente del área de grasa visceral (AGV) medida por RM y $\mathrm{PC}^{28}$.

Iribarren et $a^{29}$ recomendaron la utilización del DAS como un método sencillo y no invasivo para evaluar el riesgo de enfermedad arterial coronaria (EAC), además de la utilización del IMC, en estudios epidemiológicos. Estudios futuros deben verificar si el DAS supera ampliamente al PC y la RCC, ya que aún no hay consenso entre los estudios actuales $^{30}$.

El DAS, medido en posición supina, podría ser una alternativa interesante para evaluar la grasa visceral de pacientes que se encuentran en unidades de cuidados intensivos, ya que estos no pueden mantenerse en pie para medir el PC. Recientemente, Paolini et al también concluyeron de un estudio prospectivo multicéntrico que el DAS se considera factor independiente del riesgo de muerte en pacientes en estado crítico (odds ratio $=2,12$; intervalo de confianza del $95 \%, 1,25-3,60)^{31}$.

\section{Protocolos de medición del DAS}

Según Vasques et $\mathrm{al}^{2}$, independientemente del método utilizado para medir la grasa visceral (antropometría o técnicas de imagen), es evidente la falta de estandarización de los protocolos utilizados. Existen importantes diferencias entre los estudios con respecto a la localización anatómica y la posición del sujeto para la obtención del DAS, lo que es un factor limitante para la comparación de los datos encontrados en diferentes estudios.

Como el perfil de reserva de grasa es específico de cada sexo y grupo étnico y está influido por la edad, un único método de medición puede no ser apropiado para medir los depósitos de TA visceral de la población en su conjunto. La validación del DAS en diferentes grupos, especialmente en aquellos con exceso de peso corporal, resulta fundamental para valorar su utilidad para realizar estimaciones del TA visceral ${ }^{2,20}$.

Pese a que en los diferentes estudios citados se describe la técnica de medición del $\mathrm{DAS}^{3,18,22,25,32-35}$, en la mayoría de los estudios revisados no se hace referencia expresa al protocolo utilizado ${ }^{10,12,13,23,24,26,27,30,36-39}$.

Los estudios que citan en su metodología el equipo empleado en la medición del DAS se refieren al calibre abdominal Holtain-Kahn Abdominal Caliper ${ }^{\circledR}$ (Holtain; Dyfed Wales, Reino Unido), con brazo móvil y precisión de 0,1 cm. Además del equipo descrito, para la medición es necesaria una mesa de examen rígida ${ }^{12,13,18,21,22,24-26,30,36-40}$.

La medida del DAS se debe obtener en el milímetro más próximo, cuando el brazo móvil del compás toca levemente el abdomen, sin compresión, después del movimiento espiratorio normal. Las mediciones se realizan normalmente por duplicado, y se utiliza la media entre las dos medidas. En caso de que la diferencia entre los dos valores sea $>1 \mathrm{~cm}$, se debe descartar ambos valores y obtener dos nuevas medidas ${ }^{18,21}$.

Entre los estudios evaluados, se encontraron diferentes referencias anatómicas para la lectura del DAS: a) diámetro máximo del abdomen en el plano sagital ${ }^{12,16,27,41}$; b) talla umbilical $^{42}$; c) perímetro inferior de la región abdominal ${ }^{32}$; d) punto medio entre la última costilla y la cresta ilia$\mathrm{ca}^{10,23,29,34}$; e) nivel de la cresta iliaca ${ }^{3}$, y f) punto medio entre las crestas iliacas ${ }^{13,18,19,24}$.

El sitio anatómico más comúnmente utilizado es el punto medio entre las crestas iliacas propuesto en los estudios de $K a h n^{18,19}$, que coincide con las vértebras lumbares $L 4$ y $L 5, y$ muestra mejor correlación con el volumen de TA visceral 
obtenido por $\mathrm{TC}^{7,8}$. Sin embargo, algunos estudios han cuestionado el uso de las vértebras $L 4$ y L5 y otros sitios propuestos para la estimación del TA visceral ${ }^{43-45}$. Klein et $\mathrm{al}^{46}$ señalan que las vértebras L1 y L2 serían más apropiadas para medir el DAS.

En cuando a la posición del sujeto, el DAS se puede medir con el individuo en bipedestación ${ }^{32} \mathrm{o}$ acostado. Varios estudios apunta que el decúbito puede ser lo más adecuado ${ }^{22,47}$, pues en esta posición el TA visceral tiende a elevarse a la pared abdominal en dirección sagital y el TA abdominal subcutáneo anterior o lateral comprime el abdomen y tiende a caer hacia los lados debido a la fuerza de la gravedad. Así, se espera que el DAS medido en esta posición refleje, principalmente, el TA visceral ${ }^{47}$.

Vasques et $\mathrm{al}^{21}$ evaluaron la reproducibilidad de cuatro sitios diferentes para medir el DAS, y analizaron el poder discriminatorio de las mediciones para predecir la RI, en comparación con el índice HOMA-IR, en varones adultos $(n=190)$. Se obtuvo la medida en los siguientes sitios anatómicos: menor perímetro entre el tórax y la cadera ${ }^{32}$, mayor diámetro abdominal ${ }^{48}$, al nivel del ombligo ${ }^{42}$ y en el punto medio entre las crestas iliacas ${ }^{13}$. Las correlaciones entre los valores de HOMA-IR y las diferentes medidas del DAS fueron moderadas. De entre las cuatro medidas realizadas, la localizada a nivel del perímetro más pequeño entre el tórax y la cadera fue la que presentó mayor coeficiente de correlación con DAS $(r=0,482)$.

También se observan diferencias en el posicionamiento descrito de las rodillas durante la medición. Nordhamn et $\mathrm{al}^{31}$ demostraron que el DAS medido con las piernas dobladas es más fiable que el medido con las piernas extendidas, lo que resulta en una mejor predicción. Zamboni et $\mathrm{al}^{16}$ compararon la posición de las rodillas (extendidas frente a flexionadas) en decúbito supino, y no verificaron diferencias estadísticas entre ellas.

\section{Utilización del DAS: valor absoluto y cálculo de índices o indicadores}

La mayoría de los estudios utilizan la medida del DAS como un parámetro aislado para evaluar la adiposidad central ${ }^{3,20,21,24,28-30,38-41,50}$. Sin embargo, en algunos estudios se evaluó el IS, también conocido como índice de diámetro abdominal (IDA) 13,18,27 o índice de diámetro sagital (IDS) ${ }^{36}$, a partir de una combinación del DAS con el Pmuslo.

En gran parte de estos estudios, la combinación de medidas no mejoró la correlación del DAS con las variables estudiadas $^{10,13,23,27,36}$. Vasques et $\mathrm{al}^{22}$ encontraron más eficacia en la evaluación del riesgo de RI en varones midiendo sólo el DAS.

En contraste, Smith et $\mathrm{al}^{25}$ compararon la fuerza de la asociación de algunas medidas antropométricas con otros factores de riesgo de EAC en varones de mediana edad $(n=466)$ de diferentes grupos étnicos, y observaron que el IS tiene mayor odds ratio para el riesgo de EAC.

Kahn et $\mathrm{al}^{18}$ también evaluaron la asociación entre índices antropométricos y complicaciones coronarias en un estudio de casos y controles $(n=478)$. El IS es el índice que mejor discriminó los casos (con enfermedad isquémica del corazón) de los controles de ambos sexos. Los autores concluyeron que el IS es un método antropométrico de bajo coste, no invasivo y que se puede usar en la evaluación clínica o epidemiológica del riesgo de padecer enfermedad isquémica del corazón.

\section{Puntos de corte establecidos para el DAS y para los indicadores obtenidos con esta medida}

La mayoría de los estudios no proponen puntos de corte para la interpretación del DAS individualmente $\mathrm{e}^{10,12,18,25,26,29,33,34,36,37,40,41}$ ni para los índices calculados a partir de esta medida ${ }^{10,18,22,25,36}$.

Vasques et al $^{22}$ proponen la utilización de valores del DAS $\geq 20 \mathrm{~cm}$ para identificar el riesgo de RI en varones brasileños adultos. Valsamakis et $\mathrm{al}^{12}$ verificaron que valores de DAS $\geq 27 \mathrm{~cm}$ son capaces de predecir el SM en hombres adultos.

En un reciente estudio, realizado con adultos brasileños al objeto de comparar el DAS con el PC en su capacidad de predecir la obesidad central, también se estudiaron la sensibilidad y la especificidad de los mejores puntos de corte para el $\mathrm{DAS}^{39}$. Los autores propusieron puntos de corte específicos para identificar obesidad abdominal en cada sexo: $23,1 \mathrm{~cm}$ en varones y $20,1 \mathrm{~cm}$ en mujeres. Estos valores presentaron altas sensibilidad y especificidad (el 96 y el $86 \%$ respectivamente para los varones y el 85 y el $84 \%$ para las mujeres).

Al evaluar la fiabilidad y la validez del DAS como predictor de la grasa visceral, Sampaio et $\mathrm{al}^{13}$ observaron que los puntos de corte que ofrecen mejor combinación de alta sensibilidad (el $83 \%$ en varones y el $85 \%$ en mujeres) y alta especificidad (el $82 \%$ en varones y el $77 \%$ en mujeres) fueron $20,5 \mathrm{~cm}$ en varones y $19,3 \mathrm{~cm}$ en mujeres.

Risérus et $\mathrm{al}^{3}$ utilizaron el valor de $25 \mathrm{~cm}$ como punto de corte para identificar a varones obesos con exceso de grasa abdominal en su estudio, punto que corresponde a $100 \mathrm{~cm}$ de PC.

En un estudio sobre ancianos suecos, se proponen puntos de corte de DAS de 22,2 y $20,1 \mathrm{~cm}$ en varones y mujeres, respectivamente, para identificar a los individuos con elevado riesgo cardiometabólico ${ }^{50}$.

Al relacionar la obesidad central con la disfunción eréctil de varones de 40 años o más de la ciudad de Porto Alegre (Brasil), Riedner et $\mathrm{al}^{27}$ consideraron el valor de 0,46 como punto de corte para IS, basándose en asociaciones con factores de riesgo cardiovascular.

Hoenig ${ }^{28}$ realizó un estudio en el que se midió el DAS por RM, y observó que valores $>22,7 \mathrm{~cm}$ serían capaces de identificar SM con el $91 \%$ de sensibilidad y el $80 \%$ de especificidad. Los pacientes con DAS por encima de este punto de corte presentaron mayor RI (menor índice Quantitative Insulin Sensitivity Check [QUICK]) que aquellos con DAS por debajo $(p=0,01)$.

El análisis de curvas ROC (Receiver Operating Characteristic) se utilizó para comparar la capacidad del DAS para predecir SM definido según de los criterios del ATP-III. En la población en cuestión (una muestra de varones caucásicos y asiáticos con un IMC medio de $30,2 \pm 5,8$ y una media de edad de 50,3 \pm 13,6 años), el DAS identificó más eficientemente a los individuos con SM con un punto de corte por encima de $27 \mathrm{~cm}$, el $82,2 \%$ de sensibilidad y el $90 \%$ de especificidad $^{12}$. 
Aún hay gran divergencia entre los puntos de corte del DAS propuestos en los artículos publicados, lo que puede explicarse en parte por diferencias étnicas y de edad entre las muestras estudiadas, diferentes variables asociadas con la medida y diferentes métodos utilizados para la medición.

\section{Conclusiones}

Varios autores aconsejan utilizar la antropometría para medir el DAS, que estima la grasa visceral ${ }^{2,10,16,29,36,37,40}$, pero el pequeño número de estudios, la heterogeneidad de las poblaciones utilizadas y la falta de consenso sobre la referencia anatómica para la medida, así como puntos de corte para la clasificación, dificultan su aplicación en la práctica clínica.

Varios estudios también proponen la utilización del DAS como indicador de RI; sin embargo, es importante reconocer que esta es solamente una alternativa para identificación del riesgo $\mathrm{y}$, por lo tanto, no se lo puede considerar método de diagnóstico ${ }^{21,22}$.

La utilización del DAS combinado con otros parámetros antropométricos de la práctica clínica, como el IMC, puede ser clínicamente útil, ya que es capaz de identificar la excesiva acumulación de TA visceral incluso en individuos no obesos, que por ello tienen mayor riesgo de complicaciones metabólicas.

De este modo, son necesarios estudios epidemiológicos que tengan en cuenta los diferentes grupos étnicos para investigar la asociación entre el DAS y los factores de riesgo cardiovascular y metabólico y posibiliten la definición de puntos de corte, además de un protocolo universal para la medición del DAS.

\section{Conflicto de intereses}

Los autores expresan que no hay confictos de intereses al redactar el manuscrito.

\section{Bibliografía}

1. Kopelman PG. Obesity as a medical problem. Nature. 2000; 404:635-43.

2. Vasques ACJ, Priore SE, Rosado LEFPDL, Franceschini SDCC. Utilização de medidas antropométricas para a avaliação do acúmulo de gordura visceral. Rev Nutr. 2010;23:107-18.

3. Risérus U, Ärnlöv J, Brismar K, Zethelius B, Berglund L, Vessby B. Sagittal abdominal diameter is a strong anthropometric marker of insulin resistance and hyperproinsulinemia in obese men. Diabetes Care. 2004;27:2041-6.

4. Vasques AC, Rosado L, Rosado G, Ribeiro RDC, Franceschini S, Geloneze B. Anthropometric indicators of insulin resistance. Arq Bras Cardiol. 2010;95:1423.

5. Seidell J, Oosterlee A, Thijssen M, Burema J, Deurenberg P, Hautvast J, et al. Assessment of intra-abdominal and subcutaneous abdominal fat: relation between anthropometry and computed tomography. Am J Clin Nutr. 1987;45:7-13.

6. Pouliot $M$, Després J, Lemieux $S$, Moorjani $S$, Bouchard $C$, Tremblay A, et al. Waist circumference and abdominal sagittal diameter: Best simple anthropometric indexes of abdominal visceral adipose tissue accumulation and related cardiovascular risk in men and women. Am J Cardiol. 1994;73:460-8.

7. Kvist H, Chowdhury B, Grangard U, Tylen U, Sjostrom L. Total and visceral adipose-tissue volumes derived from measurements with computed tomography in adult men and women: predictive equations. Am J Clin Nutr. 1988;48:1351-61.

8. Sjöström L, Kvist H. Regional body fat measurements with CTscan and evaluation of anthropometric predictions. Acta Med Scand. 1988;723:169-77.

9. Despres J, Prud'homme D, Pouliot M, Tremblay A, Bouchard C. Estimation of deep abdominal adipose-tissue accumulation from simple anthropometric measurements in men. Am J Clin Nutr. 1991;54:471-7.

10. Van der Kooy K, Leenen R, Seidell JC, Deurenberg P, Visser M. Abdominal diameters as indicators of visceral fat: comparison between magnetic resonance imaging and anthropometry. $\mathrm{Br} \mathrm{J}$ Nutr. 1993;70:47-58.

11. Han TS, MCNeill G, Seidell JC, Lean ME. Predicting intraabdominal fatness from anthropometric measures: the influence of stature. Int J Obes Relat Metab Disord. 1997;21:587-93.

12. Valsamakis G, Chetty R, Anwar A, Banerjee AK, Barnett A, Kumar S. Association of simple anthropometric measures of obesity with visceral fat and the metabolic syndrome in male Caucasian and Indo-Asian subjects. Diabet Med. 2004;21:1339-45.

13. Sampaio LR, Simões EJ, Assis AMO, Ramos LR. Validity and reliability of the sagittal abdominal diameter as a predictor of visceral abdominal fat. Arq Bras Endocrinol Metabol. 2007; 51:980-6.

14. Yim JY, Kim D, Lim SH, Park MJ, Choi SH, Lee CH, et al. Sagittal abdominal diameter is a strong anthropometric measure of visceral adipose tissue in the Asian general population. Diabetes Care. 2010;33:2665-70.

15. Williamson DF, Kahn HS, Worthman CM, Burnette JC, Russell CM. Precision of recumbent anthropometry. Am J Hum Biol. 1993;5:159-67.

16. Zamboni M, Turcato E, Armellini F, Kahn HS, Zivelonghi A, Santana $\mathrm{H}$, et al. Sagittal abdominal diameter as a practical predictor of visceral fat. Int J Obes Relat Metab Disord. 1998;22:655-60

17. Radominski RB, Vezozzo DP, Cerri GG, Halpern A. O uso da ultra-sonografia na avaliação da distribuição de gordura abdominal. Arq Bras Endocrinol Metab. 2000;44:5-12.

18. Kahn HS, Austin H, Williamson DF, Arensberg D. Simple anthropometric indices associated with ischemic heart disease. J Clinl Epidemiol. 1996;49:1017-24.

19. Kahn HS, Simoes EJ, Koponen M, Hanzlick R. The abdominal diameter index and sudden coronary death in men. Am J Cardiol. 1996;78:961-4.

20. Petersson H, Daryani A, Risérus U. Sagittal abdominal diameter as a marker of inflammation and insulin resistance among immigrant women from the Middle East and native Swedish women: a cross-sectional study. Cardiovasc Diabetol. 2007;6:10.

21. Vasques ACJ, Rosado LEFPDL, Rosado GP, Ribeiro RDCL, Franceschini SDCC, Geloneze B, et al. Different measurements of the sagittal abdominal diameter and waist perimeter in the prediction of HOMA-IR. Arq Bras Cardiol. 2009;93:5118.

22. Vasques ACJ, Rosado LEFPDL, Rosado GP, Ribeiro RDCL, Franceschini SDCC, Geloneze B, et al. Predictive ability of anthropometric and body composition indicators in the identification of insulin resistance. Arq Bras Endocrinol Metabol. 2009;53:72-9.

23. Reed D, Dwyer KM, Dwyer JH. Abdominal obesity and carotid artery wall thickness. The Los Angeles atherosclerosis study. Int J Obes Relat Metab Disord. 2003;27:1546-51.

24. Nakata K, Choo J, Hopson MJ, Ueshima H, Curb JD, Shin C, et al. Stronger associations of sagittal abdominal diameter with atherogenic lipoprotein subfractions than waist circumference 
in middle-aged US white and Japanese men. Metabolism. 2010;59:1742-51.

25. Smith DA, Ness EM, Herbert R, Schechter CB, Phillips RA, Diamond JA, et al. Abdominal diameter index: a more powerful anthropometric measure for prevalent coronary heart disease risk in adult males. Diabetes Obes Metab. 2005;7: 370-80.

26. Stokic E, Brtka V, Srdic B. The synthesis of the rough set model for the better applicability of sagittal abdominal diameter in identifying high risk patients. Comput Biol Med. 2010;40:78690.

27. Riedner CE, Rhoden EL, Ribeiro EP, Fuchs SC. Central obesity is an independent predictor of erectile dysfunction in older men. J Urol. 2006;176:1519-23.

28. Hoenig MR. MRI sagittal abdominal diameter is a stronger predictor of metabolic syndrome than visceral fat area or waist circumference in a high-risk vascular cohort. Vasc Health Risk Manag. 2010;6:629-33.

29. Iribarren C, Darbinian JA, Lo JC, Fireman BH, Go AS. Value of the sagittal abdominal diameter in coronary heart disease risk assessment: cohort study in a large, multiethnic population. Am J Epidemiol. 2006;164:1150-9.

30. Mukuddem-Petersen J, Snijder MB, Van Dam RM, Dekker JM, Bouter LM, Stehouwer CD, et al. Sagittal abdominal diameter: no advantage compared with other anthropometric measures as a correlate of components of the metabolic syndrome in elderly from the Hoorn Study. Am J Clin Nutr. 2006;84:9951002.

31. Paolini JM, Mancini J, Genestal M, Gonzalez H, McKay RE, Samii $\mathrm{K}$, et al. Predictive value of abdominal obesity vs. body mass index for determining risk of intensive care unit mortality. Crit Care Med. 2010;38:1308-14.

32. Richelsen B, Pedersen SB. Associations between different anthropometric measurements of fatness and metabolic risk parameters in non-obese, healthy, middle-aged men. Int J Obes Relat Metab Disord. 1995;19:169-74.

33. Storti KL, Brach JS, FitzGerald SJ, Bunker CH, Kriska AM. Relationships among body composition measures in communitydwelling older women. Obesity. 2006;14:244-51.

34. Theorell-Haglöw J, Berne C, Janson C, Sahlin C, Lindberg E. Associations between short sleep duration and central obesity in women. Sleep. 2010;33:593-8.

35. Williamson DF, Kahn HS, Worthman CM, Burnette JC, Russell CM. Precision of recumbent anthropometry. Am J Hum Biol. 1993;5:159-67.

36. Keller C, Chintapalli K, Lancaster J. Correlation of anthropometry with CT in Mexican-American women. Res Nurs Health. 1999;22:145-53.

37. Snijder MB, Visser M, Dekker JM, Seidell JC, Fuerst T, Tylavsky $F$, et al. The prediction of visceral fat by dual-energy X-ray absorptiometry in the elderly: a comparison with computed tomography and anthropometry. Int J Obes Relat Metab Disord. 2002;26:984-93.

38. McCarthy A, Hughes R, Tilling K, Davies D, Davey Smith G, BenShlomo Y. Birth weight; postnatal, infant, and childhood growth; and obesity in young adulthood: evidence from the Barry Caerphilly Growth Study. Am J Clin Nutr. 2007;86:907-13.

39. Duarte Pimentel G, Portero-McLellan KC, Maestá N, Corrente JE, Burini RC. Accuracy of sagittal abdominal diameter as predictor of abdominal fat among Brazilian adults: a comparation with waist circumference. Nutr Hosp. 2010;25:656-61.

40. Hwu C, Hsiao C, Sheu WHH, Pei D, Tai T, Quertermous T, et al. Sagittal abdominal diameter is associated with insulin sensitivity in Chinese hypertensive patients and their siblings. J Hum Hypertens. 2003;17:193-8.

41. Kullberg J, Von Below C, Lönn L, Lind L, Ahlström H, Johansson L. Practical approach for estimation of subcutaneous and visceral adipose tissue. Clin Physiol Funct Imaging. 2007;27:148-53.

42. Ohrvall M, Berglund L, Vessby B. Sagittal abdominal diameter compared with other anthropometric measurements in relation to cardiovascular risk. Int J Obes Relat Metab Disord. 2000;24: 497-501.

43. Kamel EG, McNeill G, Han TS, Smith FW, Avenell A, Davidson L, et al. Measurement of abdominal fat by magnetic resonance imaging, dual-energy X-ray absorptiometry and anthropometry in non-obese men and women. Int J Obes Relat Metab Disord. 1999;23:686-92.

44. Shen W, Punyanitya M, Wang Z, Gallagher D, St-Onge M, Albu J, et al. Visceral adipose tissue: relations between single-slice areas and total volume. Am J Clin Nutr. 2004;80:271-8.

45. Shen W, Punyanitya M, Chen J, Gallagher D, Albu J, Pi-Sunyer $X$, et al. Visceral adipose tissue: relationships between single slice areas at different locations and obesity-related health risks. Int J Obes. 2006;31:763-9.

46. Klein S, Allison DB, Heymsfield SB, Kelley DE, Leibel RL, Nonas $C$, et al. Waist circumference and cardiometabolic risk: a consensus statement from shaping America's Health: Association for weight management and obesity prevention; NAASO, The Obesity Society; the American Society for Nutrition; and the American Diabetes Association. Am J Clin Nutr. 2007;85:1197-202.

47. Sjöström L. A computer-tomography based multicompartment body composition technique and anthropometric predictions of lean body mass, total and subcutaneous adipose tissue. Int $\mathrm{J}$ Obes. 1991;15:19-30.

48. Turcato E, Bosello O, Di Francesco V, Harris TB, Zoico E, Bissoli $\mathrm{L}$, et al. Waist circumference and abdominal sagittal diameter as surrogates of body fat distribution in the elderly: their relation with cardiovascular risk factors. Int J Obes Relat Metab Disord. 2000;24:1005-10.

49. Nordhamn K, Södergren E, Olsson E, Karlström B, Vessby B, Berglund L. Reliability of anthropometric measurements in overweight and lean subjects: consequences for correlations between anthropometric and other variables. Int J Obes Relat Metab Disord. 2000;24:652-7.

50. Risérus U, De Faire U, Berglund L, Hellénius M. Sagittal abdominal diameter as a screening tool in clinical research: cutoffs for cardiometabolic risk. J Obes [Internet]. 2010 [citado 17 Feb 2011]. Disponible en: http://www.ncbi.nlm.nih. gov/pubmed/20798888. 